\title{
EX-ORDIUM
}

\section{LA CUSTODE DELLA PROMESSA. SPUNTI PEDAGOGICI DEL RAPPORTO TRA NATURA, UOMO ED ECONOMIA NEL PENSIERO DI GALVANO DELLA VOLPE di Luca Odini}

Il contributo, nel solco di una prospettiva di pedagogia militante, si propone di analizzare, con ottica storico-critica, il rapporto tra natura, uomo ed economia nel pensiero di Galvano Della Volpe, affrontando in particolare gli studi su Marx e Rousseau, il discorso sulla disuguaglianza e la sua attualità, e l'uomo totale marxiano e la libertà comunista, per una teoria di un umanesimo positivo.

Cercheremo di mostrare come le conseguenze di questo pensiero evidenzino che nel rapporto tra economia e pedagogia, se quest'ultima non sarà consapevole del suo ruolo fondamentale di custode della promessa del progresso umano e dello sviluppo collettivo, contro la sacralizzazione della proprietà e l'istituzionalizzazione della disuguaglianza, si condannerà necessariamente a un ruolo secondario e subordinato.

The contribution aims to analyze the relationship between $\mathrm{Na}$ ture, Man and Economy in Galvano Della Volpe's thought from a critical-historical perspective, focusing in particular on the studies on Marx and Rousseau, the discourse on inequality and its relevance today, the Marxian total man and communist freedom, for a theory of a positive humanism.

The essay will try to show that in the relationship between pedagogy and economy, pedagogy needs to be aware of its function as the guardian of human progress and collective economic development against the sacralization of property and the institu- 
tionalization of inequality, lest it condemn itself to a secondary and ancillary role.

\section{Introduzione}

Il presente contributo, ponendosi nell'ottica di quella che Baldacci (2019) chiama una pedagogia militante, intende tematizzare un problema pratico e attuale, quale quello del rapporto tra economia e pedagogia, lasciandosi guidare dalle provocazioni teoretiche di Galvano della Volpe.

Appare ormai evidente come il pensiero pedagogico si trovi di fronte a un bivio che segnerà inevitabilmente il suo destino: da un lato il mercato, l'ideologia neoliberista e la sua ontologia sociale che impone universalmente il principio dell'impresa, in cui il soggetto umano è ridotto a essere un produttore e imprenditore di sé. In quest'ottica, il sapere educativo viene schiacciato da logiche eteronome che plasmano la disciplina, asservendola alle esigenze del mercato e alla mera produzione dei futuri produttori. Una visione funzionalista in cui la scuola si trasforma in una fabbrica del capitale umano e viene invasa da quella retorica dell'impresa che la trasforma in una palestra di competizione.

Basta riprendere la traccia che ci indica Baldacci (2010) per renderci conto di come quest'ideologia abbia innervato la scuola e colonizzato il sapere pedagogico, costringendolo a una funzione ancillare rispetto a logiche eteronome che rischiano di continuare a guidare e orientare i costrutti teoretici della disciplina.

Rispetto a questa possibilità e a un apparato ideologico così potente e onnipervasivo, anche solo immaginare che esista un bivio per il pensiero pedagogico appare complicato. All'interno di quest'immagine, il presente contributo vuole azzardare qualche passo su una strada diversa: utilizzando il sapere storico e un sistematico approccio critico sul reale, vogliamo mostrare come il pensiero pedagogico possa immaginarsi rivitalizzato grazie a una dialettica autonoma rispetto al pensiero economico, a condizione di essere in grado di riportare al centro i propri principi intrinseci 
che pongono come stella polare di tutte le scienze della formazione l'uomo completo, non solo il futuro produttore. La crescita dell'uomo tutto è lo scopo dell'elaborazione pedagogica, e compito di questo pensiero rimane la messa in campo di una forte forza critica in grado di smascherare ogni tipo di meccanismo che, attraverso l'educazione, voglia ridurre l'uomo da fine a mezzo.

Un rapporto dialettico tra pari dunque, in cui pedagogia ed economia possano dialogare proponendo logiche autonome che non siano ancillari o asservite a dialettiche di parte, ma in grado di proporre un orizzonte in grado di articolare paradigmi teorici di ampio respiro.

In questa che potremmo chiamare gramscianamente una lotta di trincea controegemonica, utilizzeremo l'arma del costante approccio critico, della conoscenza e dell'esperienza della storia, perché come individua Piketty (2020) «è stata la lotta per l'uguaglianza e per l'istruzione che ha permesso il progresso umano e lo sviluppo economico, e non la sacralizzazione della proprietà, della stabilità e della disuguaglianza» (p. 15).

\section{Galvano della Volpe}

In questa sede vorremmo riportare all'attenzione la figura di Galvano della Volpe, per mostrare come sia possibile affrontare, con uno sguardo diverso, autori che hanno ispirato la storia della pedagogia, proponendo una cornice alternativa rispetto a quella ontologia sociale che abbiamo individuato nelle righe precedenti.

Della Volpe, nato alla fine dell'Ottocento, morì a Roma nel 1968 dopo aver trascorso una vita di studi e insegnamento all'Università di Messina dove vinse la cattedra di Storia della Filosofia. Non si spostò mai dalla città sullo stretto non tanto per sua volontà, quanto perché il suo pensiero e la sua opera remarono incessantemente contro corrente rispetto al panorama culturale e universitario italiano. Basterebbe ricordare il suo Hegel romantico e mistico (1929) in cui diede una lettura del filosofo di Stoccarda diametralmente opposta rispetto a quella di Croce e Gentile, o anche 
lo studio su Hume (1939) o il suo Eckart o della filosofia mistica (1952), in cui sostenne che le categorie che Engels definisce le tre leggi della dialettica, erano riconducibili direttamente al neoplatonismo cristiano. Tutti questi elementi non dovevano averlo esposto sotto una buona luce presso Via delle Botteghe Oscure, e da lì, forse, il suo mancato riconoscimento ufficiale.

Un pensatore quindi di difficile collocazione, con un carattere aspro e una scrittura a volte ostica e oscura non hanno sicuramente contribuito alla divulgazione e allo studio dei suoi testi. Nonostante ciò, fu sicuramente, se non l'unico, uno dei pochi marxisti italiani a ispirare diversi studiosi tra cui Nicolao Merker, Lucio Coletti e Mario Rossi.

Di tutta la sua vasta produzione e del suo periodo di attività, per lo scopo che ci siamo prefissi, analizzeremo le opere in cui incomincia a misurarsi con il pensiero di Marx, confrontandolo con quello di Rousseau, dandoci in questo modo elementi utili per poter avere idea di una impostazione diversa di ontologia sociale rispetto a quella che stiamo vivendo, fornendoci degli elementi in grado di tematizzare e immaginare un orizzonte diverso per la persona.

\section{La critica a Roussean}

Un aspetto interessante dell'opera di Della Volpe emerge dalle critiche contro Rousseau. Se ne occupa in più scritti, ma noi ci focalizziamo in particolare sul Discorso sull'ineguaglianza (1943) e sul La teoria marxista dell'emancipazione umana (1945), opere che inaugurano il suo impegno intellettuale di sinistra.

La tesi del Discorso sull'ineguaglianza è esplicitata nella formula «eguali perché liberi e liberi perché egualì. La Rivoluzione francese e Rousseau si sono interessati principalmente della libertà (o persona), affrontando in maniera del tutto subordinata il tema dell'uguaglianza, trovandosi in questo modo a sostenere con argomenti molto deboli l'affermazione: eguali perché liberi.

Viene quindi analizzato in che modo e perché si espliciti un interesse unilaterale nel concetto tradizionale e innatistico della per- 
sona e della dignità umana singola. Uno squilibrio che può sembrare strano, ma l'obiettivo di Della Volpe è quello di criticare l'impostazione dogmatica e teologica del concetto di persona, figlio del giusnaturalismo presente in Rousseau.

Nel percorso di critica l'autore individua come il valore e la dignità dell'individuo umano vengano fondati in Rousseau su un «apriorismo dogmatico o mitico, qual è l'apriorismo platonico sinonimo di innatismo, sorge comunque la difficoltà di non poter uscire comunque dall'individualismo astratto» (Della Volpe, 1973, p. 275). Il nesso che si viene a creare appare evidente per l'autore: se l'individuo nasce con un valore universale, l'individuo (e il suo valore) si costituirà come un prius metafisico assoluto mentre il rapporto etico-sociale si costituirà come un posterius che ha fondamento nel prius metafisico. La critica a cui vuole giungere è evidente:

il rapporto etico sociale se lo si giustifica con l'amore (che presuppone una caratterizzazione aprioristico-dogmatica del "sacro" della persona umana) non potrà risolversi che in un individualismo astratto, trascendente: per cui l'individuo-persona trascende sempre il rapporto stesso (Della Volpe, 1973, pp. 275-276).

Il passaggio che individua come problematico è proprio quello che va dall'individuo empirico alla persona. Questo è evidente analizzando come si crede che avvenga questo passaggio nell'amore sessuale, che consisterebbe nell'elevare l'empirico a un valore universale, un'astrazione, concludendolo a un'ipostasi (Della Volpe, 1973, p. 276). Si tratta quindi in un certo senso di un individualismo trascendente il mondo e la società concreta che si ritrova sia nella sfera dell'amore, come sinonimo di un sentimento indistinto nella passione verso il prossimo, e a maggior ragione nella caritas cristiana, con il suo primato dello spirituale, che per Della Volpe serve a dare una evidenza essoterica all'innatismo. In Rousseau quindi, secondo l'analisi di Della Volpe, attraverso l'amore umanitario, il criterio sentimentale si spersonalizza e l'amore stesso diventa:

gusto dei puri valori, dell'universale umano, è vero altresì che questo gusto o sentimento, proprio in quanto è, con ben maggior consapevolez- 
za che non quello erotico, gusto platonico dell'universale [...] irretendosi in un individualismo astratto, particolarmente flagrante quando si tenti con esso di risolvere il problema più disperato per un romantico, quello dello Stato, come ora vedremo (Della Volpe, 1973, p. 277).

Della Volpe quindi fa seguire a questo individualismo eticometafisico di Rousseau un astrattismo per cui:

se si collega la conclusione problematica del secondo Discours - conclusione che dice che "la diseguaglianza morale, legittimata dal solo diritto positivo, è contraria al diritto naturale ogni volta che non concorra nella stessa proporzione con la diseguaglianza fisica" - se la si collega alla conclusione risolutiva del Contrat social, per cui è detto che il "patto sociale" istituisce una tale eguaglianza "morale e legittima" fra gli uomini che questi "pur potendo esser diseguali di forza e di genio, divengono tutti eguali per convenzione e di diritto" (I,9), si vedrà nettamente - nella conservazione legittima, in seno allo Stato, delle diseguaglianze "naturali" cioè immediate empiriche - ma ipostatizzate giusnaturalisticamente - si vedrà l'individualismo astratto, trascendente, di cui sopra (Della Volpe, 1973, p. 279).

Quindi è proprio questa legittimazione delle diseguaglianze empiriche degli individui che caratterizza i membri dello Stato, ed è il conseguente risultato del concepire: "l'eguaglianza in funzione della libertà (=persona) ma non viceversa: non viceversa appunto perché la persona con cui la libertà coincide è quell'individuo ipertrofico ch'è la persona originaria (del "diritto naturale"), l'ipostasi di cui sopra» (Della Volpe, 1973, p. 280). L'individuo di Rousseau si traduce quindi in un individuo astratto, e il fallimento di una formula di tipo contrattualistico come quella esaminata, parte dalle premesse stesse che sono platonico-cristiane e innatistiche che si ritrovano in questa formula. In questo senso quindi Della Volpe individua il nodo problematico del pensiero rousseauiano nel disequilibrio di libertà ed eguaglianza o giustizia:

disequilibrio che, presupponendo un concetto teologico astratto del valore o dignità (libertà) dell'individuo umano, ha il suo fondamento infi- 
ne in un narcisismo spirituale (o autocontemplazione interiore) che si traduce in narcisismo etico o individualismo astratto (Della Volpe, 1973, p. 283).

Della Volpe imposta il tema della libertà (1973, p. 285) mostrando come essa non sia un dato o un diritto con cui si nasce, ma si costituisca come un atto, un processo, un'attività particolare, storica. Per questo si pone l'eguaglianza in funzione della libertà e al tempo stesso la presuppone, ma è allo stesso modo vero come la libertà sia in funzione dell'uguaglianza e la presuppone a sua volta visto che:

tutti gli uomini, in tanto realizzano la loro libertà o attività (creatività) nel lavoro (non si tratta più di una "libertà" o un "diritto" del lavoro, come un attributo dell'ipostasi ch'è la persona originaria!), in quanto siamo realmente uguali, cioè eguali di un'eguaglianza che, appunto perché lascia sussistere come uniche differenze le differenze di sensibilità e impegno personale su cui si fonda il lavoro come attività particolare o specializzata, non è più impedita da differenze personali come dati naturali irriducibili perché ipostatizzati e assolutizzati con artificio teologico; onde le distinzioni statiche, astratte, di ceto o di classe, tradizionali, scompaiono veramente per lasciar posto soltanto a tecniche, le sole distinzioni sociali criticamente concepibili (Della Volpe, 1973, p. 285).

A questo punto, Della Volpe ha ampiamente condotto il ragionamento alla dimostrazione di come sia debole il rimedio dell'ineguaglianza umana originata dalla società impostata da Rousseau, e come si debba invece trovare un fondamento più solido.

\section{L'analisi della teoria marxista}

Affrontiamo quindi La teoria marxista dell'emancipazione umana (Della Volpe, 1945) in cui l'autore specifica come voglia far emergere dai Manoscritti economico filosofici del 1844, non tanto il Marx della politica ingabbiato in qualche sistema filosofico precostituito, quanto Marx come teorico dell'emancipazione dell'uomo. Per lo studio che ci siamo proposti ci interessa cogliere come la sua 
forza speculativa consenta di vedere sotto una luce nuova il rapporto tra natura, uomo, lavoro, economia e pedagogia, prospettandoci orizzonti diversi.

Della Volpe evidenzia come la capacità di Marx sia stata quella di individuare un pensiero emancipatorio per l'uomo in generale, non solo per il proletario ma anche per il borghese. Attorno a quest'uomo rinnovato ruoterà la concezione del lavoro come attività personale e libera. Secondo l'autore, Marx riesce nell'intento perché:

riesce - con questa filosofia - di liquidare il cristianesimo tradizionale dogmatizzato e isterilito, sia confessionale che laico (e quest'ultimo dal cosiddetto umanesimo all'illuminismo e al romanticismo), senza perdere il sostanziale insegnamento cristiano dell'eguale dignità di ogni umano individuo, anzi sviluppando questo insegnamento al massimo concepibile scientificamente; perché gli riesce insomma di conservare il cristianesimo rovesciandolo, di portare veramente in terra, nel mondo, la speranza cristiana dell'umana fraternità: donde il significato unico, incomparabile, dell'ateismo marxista (Della Volpe, 1973, p. 310).

In questo sistema che si va prefigurando muta drasticamente anche il significato del lavoro, perché si passa dal lavoro come mezzo per vivere, o sopravvivere, all'organizzazione di un lavoro inteso come primo bisogno e fine emancipatore dell'esistenza. Poche righe più avanti Della Volpe ammonisce nuovamente su un pericolo che, modificando un po' i termini in gioco, può essere paradigmatico anche per l'oggi. Ammonisce infatti come tutti gli sforzi che si tentino di fare nella mediazione tra socialismo e liberalismo, rispondano alla fine all'unica persuasione ostinata, che a volte si tramuta in fede dogmatica, che il liberalismo resti alla fin fine l'ultima parola definitiva, e l'orizzonte di riferimento a cui agganciare concetti come quello di libertà, dignità e persona (1973, p. 311). Marx, e Della Volpe con lui, sembrano invece essere pienamente convinti che il marxismo sia in grado di delineare un orizzonte non solo pratico, ma anche teoretico, in cui trovano un significato nuovo e diverso parole e ragioni morali che riguardano l'uomo, la persona, la sua dignità e la sua libertà. 
L'autore prima di proseguire la sua analisi utilizza ancora tutta la prima parte del saggio per mostrare come la morale individualistica di Rousseau derivi dalle sue premesse platonico-cristiane. Abbiamo già evidenziato il meccanismo che egli individua: dal platonismo-innatismo deriva l'intuizione dell'interiorità di Rousseau che non corrisponde alla ragione, ma al cuore, al sentimento, e da qui deriva direttamente un individualismo etico da cui deriverà allo stesso modo il suo concetto di persona.

Poste queste premesse torna nuovamente facile mostrare come, se il rapporto etico e sociale si trova fondato sull'amore umanitario, questo rapporto si risolverà necessariamente solo in un individualismo di tipo trascendente per cui la persona trascenderà sempre il rapporto stesso (Della Volpe, 1973, p. 320). L'apparato critico di Della Volpe arriva a mostrare come proprio su queste premesse si fondino il diritto di proprietà, il diritto di libertà e il diritto che potremmo chiamare naturale che individua Rousseau, ovvero delle pretese di carattere privato rovesciate come diritti razionali e naturali, da cui Rousseau costruirà il suo diritto pubblico.

Così la legittimazione delle "diseguaglianze" o differenze "naturali" o empiriche degli individui - legittimazione che consegue dalla loro giusnaturalistica ipostatizzazione richiesta dalle premesse platoniche ossia innatistiche - porta al tipo di eguaglianza-diseguaglianza che è, infatti, il risultato del concepire l'eguaglianza in funzione della libertà (=persona), ma non anche viceversa: non anche viceversa, appunto perché la persona, con cui la libertà coincide, è quella dell'individuo ipertrofico, abnorme, ch'è la persona originaria del "diritto naturale", l'ipostasi di cui sopra (Della Volpe, 1973, p. 326).

Della Volpe ha dimostrato come il perdurare della diseguaglianza umana, trovi la sua origine nel mito umanitario di Rousseau con il suo egotismo religioso, ma al tempo stesso ha mostrato i limiti ideologici di un certo tipo di emancipazione umana che Marx stesso criticherà sostituendo all'uomo di merito, l'idea dell'uomo di qualità, rompendo quei limiti ideologici assunti ormai come limiti di natura e intrinseci alla società e alla natura stessa delle cose. In questo senso la teoria marxista dell'emancipazione umana si realizza e trova la sua piena attuazione nei rapporti tra economia e etica. 
Abbiamo anche visto come Della Volpe (1973, p. 333) individui come la categoria dell'economico diventi, secondo la tradizione spiritualistica, romantica e borghese, quasi una categoria di tipo teologico e spirituale, inscrivibile alle leggi eterne insite nell'uomo. In questo senso dunque:

il rapporto di economia (=particolare) ed etica (=universale) è concepito come una riduzione della prima alla seconda, e propriamente nel senso di una spiritualizæarione dell'economico e perciò di un suo assumere un carattere individualistico astratto (Della Volpe, 1973, p. 334).

Ma qual è la soluzione che a questo punto prospetta Marx con il suo superamento positivo dell'alienazione umana? Per comprenderlo bisognerà preliminarmente esplicitare cosa sia l'uomo nella concezione di Marx. L'intento del filosofo di Treviri è quello di porre le basi per un nuovo naturalismo, o umanesimo reale, per rifondare almeno implicitamente i concetti morali; in questo senso sarà attentissimo nel tenere insieme sia gli aspetti particolari dell'uomo che quelli universali, arrivando a sostenere che la peculiarità dell'ente umano è quella di essere una unità di distinti. Marx è sempre alla ricerca dell'uomo reale, della sua materialità, cerca di rifuggire dalle astrazioni, in questo caso criticando quelle hegeliane, per rifondare il concetto di natura, e cioè di particolare. L'uomo è originariamente natura, particolare, e come ente naturale, reale, particolare, è dotato di forze naturali o vitali, delle disposizioni, delle attitudini, delle inclinazioni. Quest'uomo, in quanto ente corporeo e sensibile, è dipendente, è un ente patiens, finito, e i suoi oggetti indipendenti sono quelli dei suoi bisogni. In quanto patiens sostiene come la passione sia la forza sostanziale dell'uomo (Della Volpe, 1973, p. 338).

Ma essendo l'uomo non solo un ente particolare, naturale, è un ente che si rivolge a sé stesso, cosciente, un ente che si conferma sia nel suo essere che nel suo sapere. La sua particolarità lo rende un individuo che è un ente sociale, inteso come un ente naturale, generico e determinato. La società così non diventa una nuova astrazione. 
Tutto viene riportato, nel sistema di Marx, all'essere umano della natura in cui l'uomo come genericità si rapporta con la natura (oggettività). L'analisi marxiana prosegue specificando come la sensibilità pratica, come l'amare e il volere, non ci sono che per l'esistenza di un oggetto, per la natura divenuta umana e cioè socializzata; solamente attraverso questa strada poi universalizzata e spiritualizzata potrà acquisire un valore. In questo senso la storia dell'industria, intesa come lavoro associato, è il rapporto storico, reale, della natura con l'essere umano, ossia il rapporto reale, storico, per usare le parole di Marx, del particolare con l'universale. E qui si colloca il tema della proprietà privata, corrispondente a questa industria, che ci rende così moralmente inerti da non percepire nostra una cosa finché non la possediamo. L'uomo in questo modo, con anche i suoi sensi, si è alienato dal senso dell'avere.

Eliminando dunque il senso della proprietà privata (più in particolare della proprietà privata dei mezzi di produzione) che si nutre di lavoro e capitalizzazione, si potranno dunque emancipare in modo completo tutte le proprietà e tutti i sensi umani. In questo modo si profila il tema del comunismo come soppressione (positiva) dell'alienazione propria dell'uomo, come riappropriazione dell'uomo stesso, come riappropriazione dell'uomo per sé come uomo sociale, come uomo umano. Un comunismo quindi che si costituisce come un umanesimo e un naturalismo compiuto, che si prefigura come la soluzione dell'antagonismo fra uomo e uomo, fra uomo e natura, fra libertà e necessità (Della Volpe, 1973, p. 341).

All'interno della proprietà privata, evidenzia Della Volpe per mezzo dei testi di Marx, ogni uomo si sforza di creare all'altro un bisogno nuovo, per costringerlo a un nuovo sacrificio e per metterlo quindi di nuovo in una nuova dipendenza. Ed è qui che si svolge una potentissima traslazione di significati, ciò che io sono e ciò che io posso, non viene determinato dalla mia individualità, dalle mie potenzialità ma dal denaro, che, come una prostituta universale, permette un capovolgimento delle proprietà umane e naturali diventando il potere alienato dell'umanità. Si badi bene come nell'analisi marxiana, sia la classe possidente che quella proletaria 
sono estraniate, solo che la prima si sente a suo agio in questa estraniazione, e la intende come una forza sua medesima, mentre la seconda si sente annullata e vede annientata la possibilità di un'esistenza umana (Della Volpe, 1973, pp. 344-345).

La soppressione positiva dell'alienazione implica anche un atteggiamento che si manifesta con il materialismo pratico, o storico. La proprietà privata materiale, espressione della vita umana alienata, è la manifestazione e il frutto delle realizzazioni delle realtà dell'uomo alienato che altro non sono che modi particolari della produzione, e cadono sotto le sue leggi generali.

La soppressione dell'alienazione è quindi il ritorno dell'uomo a una vita umana, a un'esistenza umana, e cioè sociale. Della Volpe a questo punto cita Marx e i suoi Manoscritti:

[c]ome la proprietà privata non è che l'espressione sensibile del fatto che l'uomo è ad un tempo per sé oggettivo e diventa a se stesso un oggetto straniero, non umano, come la sua manifestazione di vita è l'alienazione della sua vita e la sua realizzazione è l'astrazione, una realtà straniera, così la soppressione positiva della proprietà privata, cioè l'appropriazione sensibile dell'essere umano e della vita umana, dell'uomo oggettivo, delle opere umane per e dall'uomo, non deve essere intesa unicamente nel senso del piacere immediato, esclusivo, nel senso del possesso, nel senso dell'avere. L'uomo si appropria [recupera] il suo essere generico in una guisa universale, dunque in quanto uomo totale (Marx, 1844, cit. in Della Volpe, 1973, p. 362).

Marx pone al centro l'uomo, il bisogno umano ricco, che si manifesterà anche in un nuovo modo di produzione, che sostanzierà la forza umana e si concretizzerà in una diversa forma di arricchimento. Risulterà evidente come sia esplicitata una dipendenza universale che si trasforma in una collaborazione universale degli individui. Sarà quindi proprio grazie alla comunità reale degli uomini che l'individuo, finalmente totale, potrà godere della propria libertà personale che significa attualizzazione della sua ricchezza e della sua molteplicità di interessi. Nella comunità reale quindi si può sviluppare tutta la libertà personale dell'uomo. 
Della Volpe lo sintetizza bene: l'uomo scisso in classi, nel mondo del lavoro diviso, è un uomo che perde sé stesso nella sua universalità che è genericità. La sua stessa libertà, in questo caso, non è libertà vera, bensì dipende dagli arbitri individuali, è affidata al caso, appare illusoria.

Se invece l'uomo riuscirà a stringere un legame positivo con gli altri uomini, a costruire una comunità in cui vengono annullati i gruppi privilegiati, allora sarà in grado di costruire una comunità vera in cui, mediante la sua umanità, espressa nella comunità, potrà recuperare sé stesso e la sua essenza. Il mondo ritroverà la sua vera dimensione umana proprio perché ritornerà, o diventerà, sociale. La stessa libertà, perché recuperata e assicurata attraverso la socialità, sarà una vera libertà, visto che a ognuno sarà garantita la piena realizzazione della propria individualità (Della Volpe, 1973, p. 371). L'uomo è umano, potremmo dire, in quanto è sociale, e cioè è unione compiuta di umanità e di natura.

\section{Come una conclusione}

Con il presente contributo non abbiamo voluto affrontare alcune tematiche del pensiero di Della Volpe per rivedere il suo pensiero all'interno di categorie storiografiche che sono state, seppur senza eccessi, studiate sia nel passato (Giannantoni, 1976) che nel presente (Bianchi, 2017). Abbiamo invece voluto cercare di richiamare l'attenzione su alcuni aspetti che possono aiutarci a mettere ordine nel rapporto tra economia e pensiero pedagogico.

Il primo: cogliere l'importanza di mantenere viva la possibilità che sia esistita e continui a esistere la possibilità di porre paradigmi diversi rispetto a quelli esistenti. Se l'egemonia ideologica e culturale del neoliberismo sembra ad oggi assumere le tinte di un pensiero unico che non si può scalfire, e che sembra aver innervato la natura e la società con categorie che lo rendono omnipervasivo, sappiamo anche che l'egemonia è sempre precaria (Gramsci, 1975). Per attrezzarci in questa lotta controegemonica abbiamo bisogno di segnare dei punti fermi in questa battaglia di trincea. Il primo 
potrebbe essere proprio il dovere di ricordare, il dovere della storia, e in questo caso il dovere e il compito della storia del pensiero pedagogico, di mantenere viva l'analisi su un approccio diverso che ha cercato di porre lo strumento del pensiero critico, e l'immagine dell'uomo onnilaterale, come idea di sviluppo globale della persona umana tenendo sempre presente che «tanto più comprenderemo la storia, quanto più avremo saputo partecipare consapevolmente alla vita del nostro tempo: soltanto a questo patto la storia potrà a sua volta diventare per noi maestra di vita» (Manacorda, 2012, p. 45 ).

Il secondo: attraverso questo strumento il pensiero pedagogico non può oggi non porre in essere un'analisi critica e puntuale delle condizioni reali dell'esistenza umana, per essere in grado di non smarrire gli strumenti intellettuali e di critica che possono tenere aperto un campo, un orizzonte che vada oltre quello dell'educazione piegata a un contesto neo-liberista. Piketty (2020) afferma:

sono convinto che parte del dissesto contemporaneo della democrazia derivi da un'eccessiva autonomia del sapere economico rispetto ad altre scienze sociali e al contesto civile e politico. Autonomia che è in parte conseguenza $[\ldots]$ di arrogarsi una capacità analitica e un monopolio di competenza per il quale non hanno titolo (pp. 1174-1175).

Il terzo: attraverso la coscienza storica e le argomentazioni teoriche, essere in grado di impostare il rapporto tra economia e pedagogia, e tra economia, pedagogia e politica seguendo una logica di autonomia.

Il pensiero di Della Volpe in questo senso è nato e si è sviluppato nel corso di un dibattito storico, politico ed economico che ha visto, in particolare a partire dagli anni Sessanta, la corrente riconducibile al marxismo pedagogico, contribuire ad affrontare $i$ problemi educativi sotto una luce nuova. All'interno di una tradizione certamente legata al P.C.I. si è sviluppato un approccio pedagogico alternativo che ha visto studiosi del calibro di Manacorda (1966), Bertoni Jovine (1965; 1977), Santoni Rugiu (1979), Broccoli $(1972 ; 1974 ; 1978)$ e Lombardo Radice (1976), ragionare su una scuola e su istituzioni educative che fossero in grado di emancipare 
tutto il genere umano. Evidenzia bene questo passaggio Covato (2011; 2017), mostrando quanto la critica marxista sia stata in grado di interrogare sia la pedagogia tradizionale che il dibattito epistemologico allora in corso. In tale contesto è evidente come le implicazioni di posizioni teoriche come quelle di Della Volpe e del marxismo, avrebbero condotto il dibattito a confrontarsi sul rapporto tra scuola e società, sui contenuti e metodi dei percorsi formativi, sull'idea di democrazia e sul ruolo dell'educazione nel contesto dei cambiamenti sociali e culturali con una forza propulsiva teorica e pratica non indifferente.

Allora come oggi il tema del rapporto tra democrazia ed educazione, unito a quello di problemi sempre identici ma che si palesano in maniera diversa con il passare del tempo, quali il superamento delle disuguaglianze sociali e del diritto all'educazione come chiave di emancipazione universale, sono stati al centro di un acceso dibattito che ha visto il mondo pedagogico confrontarsi anche aspramente, in nome di una nuova giustizia sociale e di una società democratica in grado di mostrare un'autonomia di pensiero e di prospettiva. Si percepisce dunque come sia essenziale, oggi, se si vuol essere in grado di scegliere consapevolmente quale strada imboccare per il futuro, ritematizzare, anche nel contesto pedagogico, quale sia il nesso che lega la natura, l'uomo, il sociale, l'educativo e l'economico, trovando il coraggio di scardinare luoghi e leggi che crediamo intrinseche alla natura stessa dell'umano ma che si nutrono invece all'interno di una chiara ideologia economica.

$\mathrm{Al}$ pensiero pedagogico dunque questo compito di riuscire a ricreare, attraverso una logica seppur conciliativa di istanze diverse, un percorso di autonomia che sia in grado di giustificare intrinsecamente un impianto diverso, in grado di mostrare che un'alternativa pedagogica c'è, è possibile ed è percorribile. Se la scuola può ancora ripensarsi e ancorarsi al modello della Costituzione (Baldacci, 2019), i modelli teorici della pedagogia hanno il dovere di tenere insieme l'analisi reale, storica e materiale dell'esperienza quotidiana che l'uomo compie, per essere in grado di proporre dei percorsi di emancipazione e di libertà in grado di realizzare la piena maturità di un uomo a tutti gli effetti onnilatera- 
le. In questo senso il pensiero pedagogico può trovare la sua forza nel riproporre il suo ruolo vigile di essere custode di una promessa: la possibilità di un arricchimento che passa attraverso la comunità degli uomini e la realizzazione delle proprie potenzialità, un uomo cacciatore, pescatore e filosofo al medesimo tempo.

\section{Bibliografia}

Baldacci M. (2017). Oltre la subalternità. Praxis e educazione in Gramsci. Roma: Carocci editore.

Baldacci M. (2019). La scuola al bivio. Mercato o democrazia? Milano: FrancoAngeli.

Bertin G.M. (1975). Educazione e alienarione. Firenze: La Nuova Italia.

Bertoni Jovine D. (1965). Storia dell'educazione popolare in Italia. Bari: Laterza.

Bertoni Jovine D. (1977). Principi di pedagogia socialista. Roma: Editori Riuniti.

Bianchi P. (2017). Una lunga fedeltà. Il marxismo di Galvano Della Volpe. Milano: Edizioni Punto Rosso.

Broccoli A. (1972). Antonio Gramsci e l'educazione come egemonia. Firenze: La Nuova Italia.

Broccoli A. (1974). Ideologia e educazione. Firenze: La Nuova Italia.

Broccoli A. (1978). Marxismo e educazione. Firenze: La Nuova Italia.

Covato C. (2011). Marxismo e educazione nell'Italia degli anni Settanta. In E.M. Bellatalla (a cura di), I sentieri della Scienza dell'educazione (pp. 222-231). Milano: FrancoAngeli.

Covato C. (2017). Democrazia e educazione. Il confronto tra marxisti e attivisti negli anni Sessanta. In L.G. Fiorucci (a cura di), John Dewey e la pedagogia democratica del Novecento (pp. 101-107). Roma: Roma.TrEPress.

Della Volpe G. (1929). Hegel romantico e mistico. Firenze: Le Monnier.

Della Volpe G. (1939). Hume o il genio dell'empirismo. Firenze: Sansoni.

Della Volpe G. (1943). Discorso sullineguaglianza. Con due saggi sull'tica dell'esisten rialismo. Roma: Ciuni.

Della Volpe G. (1945). La teoria marxista dell'emancipazione umana. Saggio sulla trasmutazione marxista dei valori. Messina: V. Ferrara.

Della Volpe G. (1946). La libertà comunista. Saggio di una critica della ragion pura pratica. Messina: V. Ferrara. 
Della Volpe G. (1949). Per la teoria di un umanesimo positivo. Studi e documenti sulla dialettica materialistica. Bologna: Zuffi.

Della Volpe G. (1952). Eckhart o della filosofia mistica. Roma: Edizioni di Storia e Letteratura.

Della Volpe G. (1957). Roussean e Marx e altri saggi di critica materialistica. Roma: Editori Riuniti.

Della Volpe G. (1964). Umanesimo positivo e emancipazione marxista. Milano: Sugar.

Della Volpe G. (1973). Opere. 6 voll. Roma: Editori Riuniti.

Giannantoni G. (1976). Il marxismo di Galvano Della Volpe. Roma: Editori Riuniti.

Gramsci A. (1975). Quaderni dal carcere. Torino: Einaudi.

Lombardo Radice L. (1976). Educazione e rivoluzione. Roma: Editori Riuniti.

Manacorda M.A. (1966). Marx e la pedagogia moderna. Roma: Editori Riuniti.

Manacorda M.A. (2008). Marx e l'educarione. Roma: Armando.

Manacorda M.A. (2012). Quel vecchio liberale del comunista Karl Marx. Roma: Aliberti Editore.

Piketty T. (2020). Capitale e ideologia. Milano: La nave di Teseo.

Santoni Rugiu A. (1979). Storia sociale dell'educazione. Milano: Principato. 